\title{
Uranyl Dicyanoaurate Coordination Polymers Through the Dimensions
}

\section{Brown ${ }^{1}$ \\ ${ }^{1}$ Simon Fraser University, Burnaby, BC mba83@sfu.ca}

The structural chemistry of uranyl $\left(\mathrm{UO}_{2}{ }^{+2}\right)$ coordination polymer chemistry has been greatly expanded by recent work, but it currently remains dominated by bridging units bearing carboxylic acids. To expand this field, we have used dicyanoaurate $\left(\mathrm{Au}(\mathrm{CN})_{2}{ }^{-}\right)$linkers in the creation of coordination polymers. Initial efforts showed an interesting array of one-dimensional coordination polymers, and we have recently completed work expanding these structures to be multidimensional using aurophilic interactions and hydrogen bonds.

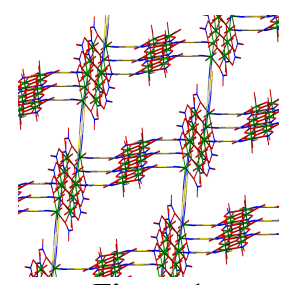

Figure 1

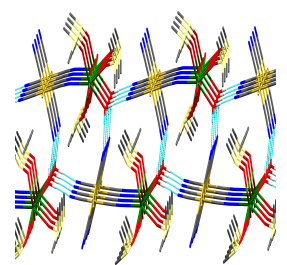

Figure 2

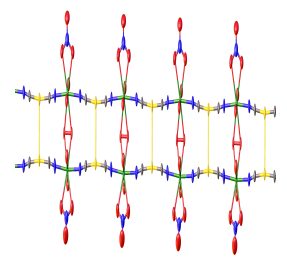

Figure 3

Acta Cryst. (2020). A76, a34 